\title{
Basic science: (AUGUST 2007)
}

1. Akli S, Van Pelt CS, Bui TY, Multani AS, Chang S, Johnson D, Tucker S, Keyomarsi K. Overexpression of the low molecular weight cyclin $E$ in transgenic mice induces metastatic mammary carcinomas through the disruption of the ARFp53 pathway. Cancer Res 2007; 67: 7212-7222.

2. Almquist M, Manjer J, Bondeson L, Bondeson AG. Serum calcium, breast cancer risk: results from a prospective cohort study of 7,847 women. Cancer Causes Control 2007; 18: 595-602.

3. Badve S, Turbin D, Thorat MA, Morimiya A, Nielsen TO, Perou CM, Dunn S, Huntsman DG, Nakshatri H. FOXA1 expression in breast cancer - correlation with luminal subtype $A$ and survival. Clin Cancer Res 2007; 13: 4415-4421.

4. Bartsch R, Wenzel C, Altorjai G, Pluschnig U, Mader RM, Gnant M, Jakesz R, Rudas M, Zielinski CC, Steger GG. Her2 and progesterone receptor status are not predictive of response to fulvestrant treatment. Clin Cancer Res 2007; 13: 4435-4439.

5. Chan MM, Lu X, Merchant FM, Iglehart JD, Miron PL. Serial transplantation of NMUinduced rat mammary tumors: a model of human breast cancer progression. Int $\mathrm{J}$ Cancer 2007; 121: 474-485.

6. Dent R, Trudeau M, Pritchard KI, Hanna WM, Kahn HK, Sawka CA, Lickley LA, Rawlinson E, Sun P, Narod SA. Triple-negative breast cancer: clinical features and patterns of recurrence. Clin Cancer Res 2007; 13: 4429-4434.

7. Drabsch $Y$, Hugo $H$, Zhang $R$, Dowhan $D H$, Miao YR, Gewirtz AM, Barry SC, Ramsay RG, Gonda TJ. Mechanism of and requirement for estrogen- regulated MYB expression in estrogenreceptor-positive breast cancer cells. Proc Natl Acad Sci USA 2007; 104: 13762-13767.

8. Frankel LB, Lykkesfeldt AE, Hansen JB, Stenvang J. Protein kinase $\mathrm{C} \alpha$ is a marker for

BCO/663/2007/JW antiestrogen resistance and is involved in the growth of tamoxifen resistant human breast cancer cells. Breast Cancer Res Treat 2007; 104: 165-179.

9. Gadir N, Jackson DN, Lee E, Foster DA. Defective TGF- $\beta$ signaling sensitizes human cancer cells to rapamycin. Oncogene 2007; 10.1038/sj.onc.1210721.

10. Gatenby RA, Smallbone K, Maini PK, Rose F, Averill J, Nagle RB, Worrall L, Gillies RJ. Cellular adaptations to hypoxia and acidosis during somatic evolution of breast cancer. Br J Cancer 2007; 97: 646-653.

11. Glass AG, Lacey JV, Carreon D, Hoover RN. Breast cancer incidence, 1980-2006: combined roles of menopausal hormone therapy, screening mammography, and estrogen receptor status. J Natl Cancer Inst 2007; 99: 1152-1161.

12. Ince TA, Richardson AL, Bell GW, Saitoh M, Godar S, Karnoub AE, Iglehart JD, Weinberg RA. Transformation of different human breast epithelial cell types leads to distinct tumor phenotypes. Cancer Cell 2007; 12: 160-170.

13. Klein $A$, Wessel R, Graessmann $M$, Juergens $M$, Petersen I, Schmutzler R, Niederacher D, Arnold N, Meindl A, Scherneck S, Seitz S, Graessmann A. Comparison of gene expression data from human and mouse breast cancers: identification of a conserved breast tumor gene set. Int J Cancer 2007; 121: 683-688.

14. Kotamraju S, Willams CL, Kalyanaraman B. Statin-induced breast cancer cell death: role of inducible nitric oxide and arginase-dependent pathways. Cancer Res 2007; 67: 7386-7394.

15. Kuo PL, Chen CY, Hsu YL. Isoobtusilactone A induces cell cycle arrest and apoptosis through reactive oxygen species/apoptosis signalregulating kinase 1 signaling pathway in human breast cancer cells. Cancer Res 2007; 67: 7406-7420.

16. Kuorelahti A, Rulli S, Huhtaniemi I, Poutanen M. Human chorionic gonadotropin (hCG) up-regulates $w n t 5 b$ and $w n t 7 b$ in the mammary gland, and 
hCGß transgenic female mice present with mammary gland tumors exhibiting characteristics of the wnt/B-catenin pathway activation. Endocrinology 2007; 148: 3694-3703.

17. Lahusen T, Fereshteh M, Oh A, Wellstein A, Riegel AT. Epidermal growth factor receptor tyrosine phosphorylation and signaling controlled by a nuclear receptor coactivator, amplified in breast cancer 1. Cancer Res 2007; 67: 7256-7265.

18. Lu D, Sivaprasad U, Huang J, Shankar E, Morrow S, Basu A. Protein kinase C-epsilon protects MCF-7 cells from TNF-mediated cell death by inhibiting Bax translocation. Apoptosis 2007; 12: 1893-1900.

19. Nangia-Makker P, Tait L, Shekhar MPV, Palomino E, Hogan V, Piechocki MP, Funasaka T, Raz A. Inhibition of breast tumor growth and angiogenesis by a medicinal herb: Ocimum gratissimum. Int J Cancer 2007; 121: 884-894.

20. Ntukidem NI, Nguyen AT, Stearns V, Rehman M, Schott A, Skaar T, Jin Y, Blanche P, Li L, Lemler S, Hayden J, Krauss RM, Desta Z, Flockhart DA, Hayes DF. Estrogen receptor genotypes, menopausal status, and the lipid effects of tamoxifen. Clin Pharmacol Ther 2007; 10.1038/ sj.clpt.6100343.

21. Razanajaona D, Joguet S, Ay AS, Treilleux I, Goddard-Leon S, Bartholin L, Rimokh R. Silencing of FLRG, an antagonist of activin, inhibits human breast tumor cell growth. Cancer Res 2007; 67: 7223-7229.

22. Song RXD, Zhang ZG, Chen YC, Bao YD, Santen RJ. Estrogen signaling via a linear pathway involving insulin-like growth factor I receptor, matrix metalloproteinases, and epidermal growth factor receptor to activate mitogen-activated protein kinase in MCF-7 breast cancer cells. Endocrinology 2007; 148: 4091-4101.

23. Tamimi RM, Byrne C, Colditz GA, Hankinson $\mathrm{SE}$. Endogenous hormone levels, mammographic density, and subsequent risk of breast cancer in postmenopausal women. I Nat/ Cancer Inst 2007; 99: 1178-1187.

24. Treszezamsky AD, Kachnic LA, Feng ZH, Zhang JR, Tokadjian C, Powell SN. BRCA1and BRCA2-deficient cells are sensitive to etoposide-induced DNA double-strand breaks via topoisomerase II. Cancer Res 2007; 67: 7078-7081.

25. Wendt MK, Cooper AN, Dwinell MB. Epigenetic silencing of CXCL12 increases the metastatic potential of mammary carcinoma cells. Oncogene 2007; 10.1038/sj.onc.1210751.

26. Williams C, Edvardsson K, Lewandowski SA, Stroem A, Gustafsson JA. A genome-wide study of the repressive effects of estrogen receptor $\beta$ on estrogen receptor $\alpha$ signaling in breast cancer cells. Oncogene 2007; 10.1038/ sj.onc.1210712.

27. Zheng AP, Kallio A, Harkonen P. Tamoxifeninduced rapid death of MCF-7 breast cancer cells is mediated via extracellularly signalregulated kinase signaling and can be abrogated by estrogen. Endocrinology 2007; 148: 3931-3931.

Prepared by

$R$ Sutherland

Cancer Research Program

Garvan Institute of Medical Research

Darlinghurst, NSW, Australia 